\title{
ROBUSTNESS IN THE PRESENCE OF JOINT PARAMETRIC UNCERTAINTY AND UNMODELED DYNAMICS
}

\author{
Michael K.H. Fan and André L. Tits \\ Electrical Engineering Department and Systems Research Center \\ University of Maryland, College Park, MD 20742 \\ John C. Doyle \\ Electrical Engineering Department \\ California Institute of Technology, Pasadena, CA 91125
}

\begin{abstract}
It is shown that, in the case of joint real parametric and complex uncertainty, Doyle's structured singular value can be obtained as the solution of a smooth constrained optimization problem. While this problem may have local maxima, an improved computable upper bound to the structured singular value is derived, leading to a sufficient condition for robust stability and performance.
\end{abstract}

\section{Introduction}

An inherent tradeoff in modeling is between fidelity and simplicity. It is desirable to have models which closely match reality, yet are still easy to analyze. This tradeoff arises in modeling uncertainty. For example, a single norm-bounded perturbation makes analysis simple but may be too conservative. Introducing more structure may improve the model fidelity but typically complicates the analysis. It is often very natural to model uncertainty with real perturbations when, for example, the real coefficients of a differential equation model are uncertain. It is important, however, to remember that such parametric variations are in a model, not in the physical system being modeled. Models with real parametric uncertainty are used because, in principle, they allow more accurate representation of some systems.

The structured singular value (SSV or $\mu$ ) was introduced to study structured uncertainty in linear models [1]. The SSV, as originally formulated, gives a precise characterization of robust stability and performance, in an $H_{\infty}$ sense, for systems with complex uncertainty. Complex perturbations are typically used to represent uncertainty due to unmodeled dynamics, or to "cover" the variations produced by several real parameters. In the $\mu$ framework, complex uncertain blocks also arise for problems of robust performance [2].

This paper focuses on computation of $\mu$ for mixed real parametric and complex uncertainty. Although computation of $\mu$ for complex perturbations is nontrivial and there are important outstanding issues to be resolved, substantial progress has been made and $\mu$ is being applied routinely to large engineering problems. Computation of $\mu$ for real perturbations is fundamentally more difficult than for complex perturbations.
The major issues in computing $\mu$, or its equivalent, are the generality of the problem description, the exactness of analysis, and the ease of computation. Existing methods for real perturbations provide for two of these. A general and, in principle, exact method is a brute force global search using a grid of parameter values (e.g. $[3,4])$. This inevitably involves an exponential growth in computation as a function of the number of parameters, and taking fewer grid points to avoid this gives up exactness. Progress is being made in reducing the computational burden of exact methods [5-7], but nothing suggestive of polynomial-time algorithms is available.

An approach to obtaining exact results with modest computation is to restrict the problem description. The best example is Kharitonov's celebrated result for polynomials with coefficients in intervals [8]. Unfortunately, it is almost impossible to find models with any engineering motivation that fit the allowable problem description. Again, progress is being made in this direction by allowing more general uncertainty descriptions at the expense of more computation (e.g. [9-11]).

The approach taken in this paper could be characterized as being very general and computationally attractive, but potentially inexact. Following the methods developed for $\mu$ in the case of complex perturbations, the main idea is to get upper and lower bounds using local search methods which are computationally inexpensive, but may fail to find global solutions. One then seeks to prove that the local methods yield global solutions, or that the bounds one gets are tight enough to be of value in problems of interest. This strategy has been very successful in the case when all perturbations are complex and appears to have promise for the general case as well, although it is clear that the latter is much more challenging.

\section{Notation and Preliminaries}

Throughout the paper, given any square complex matrix $M$, we denote by $\bar{\sigma}(M)$ its largest singular value, by $\bar{M}$ its complex conjugate and by $M^{H}$ its complex conjugate transpose, and we let $\rho_{R}(M)=\max \{|\lambda|: \lambda$ is a real eigenvalue of $M\}$, with $\rho_{R}(M)=0$ if $M$ has no real eigenvalue. Given any complex vector $x, x^{H}$ indicates its complex conjugate transpose and $\|x\|$ its Euclidean norm. 
The empty set will be denoted by 0 . Finally, while $j$ will be used as a running index, $j$ will denote $\sqrt{-1}$.

Given an $n \times n$ complex matrix $M$ and three nonnegative integers $m_{r}, m_{c}$, and $m_{C}$, with $m:=m_{r}+m_{c}+$ $m_{C} \leq n$, a block structure $K$ of dimensions $\left(m_{r}, m_{c}, m_{C}\right)$ associated with $M$ is an $m$-tuple of positive integers

$$
\begin{gathered}
\mathcal{K}=\left(k_{1}, \ldots, k_{m_{r}} ; k_{m_{r}+1}, \ldots, k_{m_{r}+m_{e}} ;\right. \\
\left.k_{m_{r}+m_{c}+1}, \ldots, k_{m}\right)
\end{gathered}
$$

such that $\sum_{q=1}^{m} k_{q}=n$. Given a block structure $K$, consider the family of block diagonal $n \times n$ matrices

$$
\begin{gathered}
X_{K}=\left\{\Delta = \text { block } \operatorname { d i a g } \left(\delta_{1}^{r} I_{k_{1}}, \ldots, \delta_{m_{r}}^{r} I_{k_{m r}},\right.\right. \\
\left.\delta_{1}^{c} I_{k_{m_{r}+1}}, \ldots, \delta_{m_{c}}^{c} I_{k_{m_{r}+m_{c}}}, \Delta_{1}^{C}, \ldots, \Delta_{m_{c}}^{C}\right): \\
\left.\delta_{q}^{r} \in \mathbb{R}, \delta_{q}^{c} \in \mathbb{C}, \Delta_{q}^{C} \in \mathbb{C}^{k_{m r+m_{c}+q} \times k_{m_{r}+m_{c}+q}}\right\},
\end{gathered}
$$

and, for any nonnegative real number $\delta$, let

$$
X_{X}(\delta)=\left\{\Delta \in X_{X}: \bar{\sigma}(\Delta) \leq \delta\right\}
$$

The 'repeated real scalar' blocks $\delta_{q}^{r} I_{k_{q}}$ corresponds to parametric uncertainty, one 'repeated complex scalar' block $\delta_{q}^{c} I_{k_{r}+q}$ can be used to represent frequency (see [2] for details; several such blocks are introduced here mostly for the sake of uniformity) and the 'full complex' blocks $\Delta^{C}$ correspond to unmodeled dynamics.

Definition 1.1.[1]The structured singular value $\mu_{K}(M)$ of a complex $n \times n$ matrix $M$ with respect to block structure $K$ is the positive number $\mu$ having the property that

$$
\operatorname{det}(I-\Delta M) \neq 0 \text { for all } \Delta \in X_{X}(\delta)
$$

if, and only if, $\delta \mu<1$. In other words, $\mu_{\kappa}(M)$ is 0 if there is no $\Delta$ in $X_{X}$ such that $\operatorname{det}(I-\Delta M)=0$, and

$$
\left(\min _{\Delta \in \bar{X}_{K}}\{\bar{\sigma}(\Delta): \operatorname{det}(I-\Delta M)=0\}\right)^{-1}
$$

otherwise.

Directly from Definition 1.1 , it is easily shown that

$$
\rho_{R}(M) \leq \mu_{K}(M) \leq \bar{\sigma}(M)
$$

and that, for all $U \in U_{K}, D \in D_{K}$,

$$
\mu_{K}(M)=\mu_{K}\left(D M U D^{-1}\right)
$$

where

$$
u_{X}=\left\{U \in X_{K}: U U^{H}=I\right\}
$$

and

$$
\begin{gathered}
D_{K}=\left\{\text { block } \operatorname { d i a g } \left(D_{1}, \ldots, D_{m_{r}+m_{e}}, d_{1} I_{k_{m_{r}+m_{e}+1}}, \ldots,\right.\right. \\
\left.\left.d_{m_{c}} I_{k_{m}}\right): 0<D_{q}=D_{q}^{H} \in \mathbf{C}^{k_{q} \times k_{q}}, d_{q} \in \mathbf{R}\right\} .
\end{gathered}
$$

Combining these two sharpens (1.3) to $\dagger$

$$
\max _{U \in \mathcal{U}_{K}} \rho_{R}(M U) \leq \mu_{K}(M) \leq \inf _{D \in D_{K}} \bar{\sigma}\left(D M D^{-1}\right)
$$

For the purely complex case $\left(m_{r}=0\right)$, the $\rho_{R}$ inequality is always an equality and the $\bar{\sigma}$ inequality is an equality when $2 m_{c}+m_{C} \leq 3[1,12]$. The $\rho_{R}$ expression has nonglobal local maxima while every local minimum of the $\bar{\sigma}$ expression is global. Extensive computational experience has suggested that it is easy to obtain $U \in U_{K}$ making $\rho_{R}(M U)$ close to the latter, even when $m_{C} \gg 1$. These bounds formed the basis for early computational approaches to $\mu$ for $m_{r}=0$, because local search methods could be used to make the bounds reasonably tight $[1,13,14]$.

Unfortunately, when $m_{r}>0$ the bounds in (1.5) may be arbitrarily far off, even for problems with engineering motivation. In [2] an improved upper bound was obtained but no practical way to compute it was given. This paper provides an alternative maximization to the $\rho_{R}$ expression which is equal to $\mu_{K}(M)$ at its global maximum. The new expression suggests a geometric interpretation based on the concept of 'multiform numerical range'. Also, an upper bound mathematically equivalent to the one in [2] is obtained, but with much better computational properties.

The balance of the paper is organized as follows. In Section 2 , it is shown that $\mu$ can be obtained as the optimal value of a smooth constrained optimization problem. Geometric interpretation of this result is discussed in Section 3. The framework of Sections 2 and 3 is used in Section 4 to derive a computable upper bound on $\mu$ that improves on the $\bar{\sigma}$ expression in (1.5). In Section 5 the new bound is shown to be mathematically equivalent to that of [2].

\section{A Smooth Optimization Problem}

Definition 1.1 suggests that one consider matrices $\Delta \in X_{K}$ such that, for some nonzero $x$

$$
\Delta M x=x
$$

$\dagger$ Note that, if $m_{r}=0, \rho_{R}$ can be equivalently replaced by the spectral radius $\rho$. 
Without loss of generality, $x$ has unit length, i.e.,

$$
x \in \partial B:=\left\{x \in \mathbb{C}^{n}:\|x\|=1\right\}
$$

In view of the structure of $\Delta,(2.1)$ imposes some constraints on 'subvectors' of $x$, corresponding to $x$ being split according to structure $K$. To make this precise, we consider projection matrices $P_{q}, q=1, \ldots, m$, defined by

$$
P_{q}=\text { block diag }\left(O_{k_{1}}, \ldots, O_{k_{q-1}}, I_{k_{q}}, O_{k_{q}+1}, \ldots, O_{k_{m}}\right)
$$

and their respective $i$ th block rows $Q_{q}, q=1, \ldots, m$,

$$
\begin{gathered}
Q_{q}=\text { block row }\left(O_{k_{1} \times k_{q}}, \ldots, O_{k_{q}-1 \times k_{q}}, I_{k_{q}},\right. \\
\left.O_{k_{q}+1} \times k_{q}, \ldots, O_{k_{m} \times k_{q}}\right)
\end{gathered}
$$

Here, for any positive integers $k, k^{\prime}, I_{k}$ is the $k \times k$ identity matrix, $O_{k}$ the $k \times k$ zero matrix, and $O_{k \times k^{\prime}}$ the $k \times k^{\prime}$ zero matrix. If $m_{r}=m_{c}=0$, the constraints on $x$ implied by (2.1) are simply

$$
\Delta_{q} Q_{q} M x=Q_{q} x, \quad q=1, \ldots, m_{C} \quad\left(m_{r}=m_{c}=0\right)
$$

and, in order for this to be achieved for some $\Delta, \bar{\sigma}(\Delta) \leq$ $\delta$, it is necessary and sufficient that

$$
\delta\left\|P_{q} M x\right\| \geq\left\|P_{q} x\right\|
$$

Thus $\mu_{K}(M)=1 / \delta^{*}$, with $\delta^{*}$ the smallest $\delta$ for which such $x$ exists. Letting $=1 / \delta$ one gets $\dagger$

$\mu_{K}(M)=\max _{\substack{x \in \delta_{0} \\ \theta \geq 0}}\left\{\theta\left\|P_{q} M x\right\| \geq \theta\left\|P_{q} x\right\|, \quad q=1, \ldots, m_{C}\right\}$ $\left(m_{r}=m_{c}=0\right)(2.2)$

which accounts for the possibility that there be no $\Delta \epsilon$ $X_{X}$ satisfying $\operatorname{det}(I-\Delta M)=0$, in which case $\mu_{K}(M)=$ 0 .

When repeated scalar blocks are present, more constraints need be taken into account. For the sake of simplicity, consider a structure $K$ involving only one block of each type, i.e., $m_{r}=m_{c}=m_{C}=1, K=\left(k_{1} ; k_{2} ; k_{3}\right)$. Equation (2.1) now implies additional constraints besides those in (2.2), namely (if $x_{i} \neq 0, i=1, \ldots, k_{1}$ )

$$
\frac{(M x)_{i}}{x_{i}}=\frac{(\overline{M x})_{j}}{\bar{x}_{j}}, \quad i, j=1, \ldots, k_{1}
$$

(including the case $i=j$ ), and

$$
\frac{(M x)_{i}}{x_{i}}=\frac{(M x)_{j}}{x_{j}}, \quad i, j=k_{1}+1, \ldots, k_{1}+k_{2}
$$

as these are equivalent to the existence of $s \in \mathbf{R}$ (resp. $s \in \mathbf{C})$ such that

$\zeta(M x)_{i}=x_{i},-i=1, \ldots, k_{1} \quad$ (resp. $i=k_{1}+1, \ldots, k_{2}$ ) .

\footnotetext{
$\dagger$ A related result was obtained in [14].
}

Also, under the constraints in (2.2), one must have $\varsigma \leq$ $1 / \theta$. The above is formalized in the next lemma.

Lemma 2.1. Let $\theta>0, x \in \mathbb{C}^{n}$, and let $P=$ $\operatorname{diag}\left(p_{1}, \ldots, p_{n}\right)$, with $p_{i}=1, i=i_{0}, i_{0}+1, \ldots, i_{\ell}, p_{i}=0$ elsewhere, for some $i_{0}, i_{l} \in\{1, \ldots, n\}$. Suppose that

$$
\|P M x\| \geq \theta\|P x\|
$$

Then (i)

$$
x_{i}(\overline{M x})_{j}=\bar{x}_{j}(M x)_{i}, \quad i, j=i_{0}, \ldots, i_{\ell}
$$

if, and only if, for some $\delta \in[-1,1]$,

$$
\delta(M x)_{i}=\theta x_{i}, \quad i=i_{0}, \ldots, i_{\ell}
$$

and (ii)

$$
x_{i}(M x)_{j}=x_{j}(M x)_{i}, \quad i, j=i_{0}, \ldots, i_{\ell}
$$

if, and only if, (2.5) holds for some $\delta \in C,|\delta| \leq 1$.

Thus the vector $x$ should be constrained to lie in the subset $S_{X}(M)$ of $\mathbf{C}^{n}$ given by

$$
\begin{aligned}
& S_{X}(M)=\{x \in \partial B: \\
& x_{i}(\overline{M x})_{j}=\bar{x}_{j}(M x)_{i}, \quad i, j=1, \ldots, k_{1} ; \\
& \left.x_{i}(M x)_{j}=x_{j}(M x)_{i}, \quad i, j=k_{1}+1, \ldots, k_{1}+k_{2}\right\} .
\end{aligned}
$$

The above is still valid for the general structure (1.1) with $S_{K}(M)$ now given by

$$
\begin{aligned}
& S_{K}(M)=\{x \in \partial B: \\
& x_{i}(\overline{M x})_{j}=\bar{x}_{j}(M x)_{i}, \quad(i, j) \in \bigcup_{q=1}^{m_{r}} J_{q} \times J_{q} ; \\
& \left.x_{i}(M x)_{j}=x_{j}(M x)_{i}, \quad(i, j) \in \bigcup_{q=m_{r}+1}^{m_{r}+m_{e}} J_{q} \times J_{q}\right\},
\end{aligned}
$$

where, for $q=1, \ldots, m_{r}+m_{c}$, the index set $J_{q}$ is defined by

$$
J_{q}=\left\{\sum_{p=1}^{q-1} k_{p}+1, \sum_{p=1}^{q-1} k_{p}+2, \ldots, \sum_{p=1}^{q} k_{p}\right\}
$$

Equation (2.2) is thus generalized as follows.

Theorem 2.1. For any matrix $M$ and associated structure $K, \mu_{K}(M)=0$ if $S_{K}(M)=0$, and

$$
\mu_{X}(M)=\max _{\substack{x \in S_{K}(M) \\ \theta \geq 0}}\left\{\theta:\left\|P_{q} M x\right\| \geq \theta\left\|P_{q} x\right\|, q=1, \ldots, m\right\}
$$

otherwise.

Formula (2.7) for $\mu_{X}(M)$ has some definite computational advantages over the formula defining $\mu_{\chi}(M)$ in Definition 2.1. The number of variables is limited, the objective and constraints are inexpensive to evaluate and, 
after squaring all the norms, objective and constraints become smooth. However, again, (2.7) may have local maxima which are not global and it is not clear whether the global maximum can be easily obtained.

\section{Geometric Interpretation}

Formula (2.7) leads to a characterization of the structured singular value in terms of the multiform numerical range of some matrices. The multiform numerical range (or $m$-form numerical range) of an $m$-tuple of $n \times n$ Hermitian matrices $A_{1}, \ldots, A_{m}$ is the set

$$
W_{K}\left(A_{1}, \ldots, A_{m}\right)=\{f(x): x \in \partial B\}
$$

where $f: \mathbf{C}^{n} \rightarrow \mathbb{R}^{m}$ has components

$$
f_{q}(x)=x^{H} A_{q} x, \quad q=1, \ldots, m .
$$

First suppose that $m_{r}=m_{c}=0$. For $\alpha \in \mathbf{R}$, let

$$
A_{q}(\alpha)=\alpha P_{q}-M^{H} P_{q} M, \quad q=1, \ldots, m_{C},
$$

and let $W_{X}(\alpha)$ be the $m$-form numerical range $W_{K}\left(A_{1}(\alpha)\right.$, $\left.\ldots, A_{m}(\alpha)\right)$. Then $\dagger$

with

$$
\begin{array}{r}
\mu_{K}(M)=\inf _{\alpha \geq 0}\left\{\sqrt{\alpha}: 0 \notin W_{K}(\alpha)+\mathbf{R}_{+}^{m}\right\} \\
\left(m_{r}=m_{c}=0\right)(3.2)
\end{array}
$$

$$
\mathbf{R}_{+}^{m}=\left\{v \in \mathbf{R}^{m}: v_{q} \geq 0, \quad q=1, \ldots, m\right\} .
$$

This follows rather directly if one rewrites the constraints in (2.7) as

$$
x^{H} A_{q}\left(\theta^{2}\right) x \leq 0, \quad q=1, \ldots, m_{C} .
$$

As a first step toward generalizing (3.2) to the case when $m_{r} \neq 0$ (we will still assume $m_{c}=0$ ), we now give an equivalent characterization of $S_{\mathcal{K}}(M)$. Again, to simplify notation, we let $m_{r}=m_{C}=1$. For this we make use of $n \times n$ matrices $E^{i j}, i, j=1, \ldots, k_{1}$, defined by

$$
\left(E^{i j}\right)_{k \ell}= \begin{cases}1 & \text { if } k=i \text { and } \ell=j \\ 0 & \text { otherwise. }\end{cases}
$$

Proposition 3.1.

$$
\begin{gathered}
S_{\mathcal{K}}(M)=\{x \in \partial B: \\
\left.x^{H} E^{i j} M x=x^{H} M^{H} E^{i j} x, \quad i, j=1, \ldots, k_{1}\right\} . \\
\left(m_{c}=0\right)
\end{gathered}
$$

Consider now the family of $k_{1}^{2}$ elementary Hermitian matrices

$$
\begin{aligned}
\mathcal{E}= & \left\{\left(E^{i j}+E^{j i}\right): i, j=1, \ldots, k_{1}, i \leq j\right\} \\
& \cup\left\{j\left(E^{i j}-E^{j i}\right): i, j=1, \ldots, k_{1}, i<j\right\} .
\end{aligned}
$$

$\dagger$ A related result was obtained in [15].
$S_{X}(M)$ can be written as

$$
S_{X}(M)=\left\{x \in \partial B: x^{H} E M x=x^{H} M^{H} E x, \forall E \in \mathcal{E}\right\} .
$$$$
\left(m_{\varepsilon}=0\right)(3.3)
$$

Next, for $q=1,2$ and $\alpha \in \mathbf{R}$, let

$$
A_{q}(\alpha)=\alpha P_{q}-M^{H} P_{q} M
$$

and for $q=1, \ldots, k_{1}^{2}$, let

$$
A_{2+q}(\alpha)=j\left(E_{q} M-M^{H} E_{q}\right)
$$

where $E_{1}, \ldots, E_{k_{1}^{2}}$ are the elements of $\varepsilon$, taken in some arbitrary order, and where the argument $\alpha$ is used for the sake of uniformity of notation. Then $A_{q}(\alpha)=A_{q}(\alpha)^{H}$, $q=1, \ldots, 2+k_{1}^{2}$ and $\mu_{X}(M)=0$ if $S_{K}(M)=\emptyset$, and

$$
\begin{aligned}
\mu_{X}(M)=\max _{\substack{\sum_{G g} \\
\alpha \geq 0}}\left\{\sqrt{\alpha: x^{H} A_{q}(\alpha) x \leq 0, q}=1,2 ;\right. \\
\left.x^{H} A_{q}(\alpha) x=0, q=3, \ldots, 2+k_{1}^{2}\right\} \\
\left(m_{c}=0\right)
\end{aligned}
$$

otherwise. Denoting by $W_{K}(\alpha)$ the $\left(2+k_{1}^{2}\right)$-form numerical range associated with $A_{1}(\alpha), \ldots, A_{2+k_{1}^{2}}(\alpha)$, i.e.,

$$
W_{K}(\alpha)=\left\{v \in \mathbf{R}^{2+k_{1}^{2}}:\right.
$$

$\exists x \in \partial B$ s.t. $\left.v_{q}=x^{H} A_{q}(\alpha) x, \quad q=1, \ldots, 2+k_{1}^{2}\right\}$,

we obtain the following result, to be compared with (3.2). Here the set $P_{2} \subset \mathbf{R}^{2+k_{1}^{2}}$ is defined by

$$
\begin{aligned}
P_{2}=\left\{v \in \mathbf{R}^{2+k_{1}^{2}}: v_{q}\right. & \geq 0, q=1,2 ; \\
v_{q} & \left.=0, q=3, \ldots, 2+k_{1}^{2}\right\} .
\end{aligned}
$$

\section{Theorem 3.1.}

$$
\mu_{K}(M)=\inf _{\alpha \geq 0}\left\{\sqrt{\alpha}: 0 \notin W_{X}(\alpha)+P_{2}\right\} . \quad\left(m_{c}=0\right)
$$

Again, this is easily generalized to the general structure (1.1) with $m_{c}=0$ by defining

$$
\begin{aligned}
\mathcal{E}= & \left\{\left(E^{i j}+E^{j i}\right):(i, j) \in \bigcup_{q=1}^{m_{r}} J_{q} \times J_{q}, i \leq j\right\} \\
& \bigcup\left\{j\left(E^{i j}-E^{j i}\right):(i, j) \in \bigcup_{q=1}^{m_{r}} J_{q} \times J_{q}, i<j\right\},
\end{aligned}
$$

defining $W_{K}(\alpha)$ accordingly and substituting $P_{m}(m=$ $\left.m_{r}+m_{C}\right)$ for $P_{2}$ with

$$
\begin{aligned}
P_{m}= & \left\{v \in \mathbf{R}^{m+\sum_{q=1}^{m r} k_{q}^{2}}:\right. \\
& v_{q} \geq 0, q=1, \ldots, m ; \\
& \left.v_{q}=0, q=m+1, \ldots, m+\sum_{q=1}^{m_{r}} k_{q}^{2}\right\} .
\end{aligned}
$$


Let us now define, for any $\alpha \in \mathbf{R}$,

$$
c_{K}(\alpha)=\min \left\{\|v\|: v \in W_{K}(\alpha)+P_{m}\right\}
$$

and let us consider the following algorithm.

Algorithm 3.1. (Computation of $\mu_{K}(M)$ when $m_{c}=0$ )

Step 0 . Set $\alpha_{0}=\bar{\sigma}^{2}(M)$ and $k=0$.

Step 1. Set $\alpha_{k+1}=\alpha_{k}-c_{K}\left(\alpha_{k}\right)$.

Step 2. Set $k=k+1$ and go to Step 1 .

The following can be proved.

Theorem 3.2. The sequence $\left\{\alpha_{k}\right\}$ generated by Algorithm 3.1 is monotone nonincreasing and

$$
\lim _{k \rightarrow \infty} \sqrt{\alpha_{k}}=\mu_{X}(M)
$$

Algorithm 3.1 can be implemented whenever $W_{K}(\alpha)$ is convex. Since the multiform numerical range of no more than 3 matrices is always convex (provided these matrices have size at least $3 \times 3)$ [16-18], this will always be the case when $m_{r}+m_{C}+\sum_{1}^{m_{r}} k_{q}^{2} \leq 3$, i.e., in the case of 3 or fewer complex blocks $\left(m_{r}=0, m_{C} \leq 3\right)$ or 1 real scalar block and one or no complex blocks $\left(m_{r}=1, k_{1}=\right.$ $\left.1, m_{C} \leq 1\right)$.

\section{A Computable Upper Bound}

Consider the family of Hermitian matrices

$$
\begin{gathered}
\mathcal{G}_{K}=\left\{\operatorname { b l o c k } \operatorname { d i a g } \left(G_{1}, \ldots, G_{m_{r}},\right.\right. \\
\left.O_{k_{m_{r}+1}}, \ldots, O_{k_{m_{r}+m_{e}}}, O_{k_{m_{r}+m_{e}+1}}, \ldots, O_{k_{m}}\right) ; \\
\left.G_{q}=G_{q}^{H} \in \mathbf{C}^{k_{q} \times k_{q}}, \quad q=1, \ldots, m_{r}\right\} .
\end{gathered}
$$

Clearly, for any $G \in \mathcal{G}_{X}, j\left(G M-M^{H} G\right)$ is Hermitian. Also, in view of (3.3) and (3.4),

$$
x^{H}\left(G M-M^{B} G\right) x=0 \text { for all } x \in S_{K}(M) .
$$

This leads to the following result which relates $\mu_{K}(M)$ and $\bar{\sigma}(M)$ to the quantities

$$
\eta_{K}(M)= \begin{cases}0 & \text { if } S_{X}(M)=\emptyset \\ \max _{x \in S_{K}(M)}\|M x\| & \text { otherwise }\end{cases}
$$

and

$\nu_{K}(M)=\sqrt{\max \left\{0, \inf _{G \in \mathcal{g}_{K}} \bar{\lambda}\left[M^{H} M+j\left(G M-M^{H} G\right)\right]\right\}}$.

Proposition 4.1. For any matrix $M$ and associated structure $K$,

$$
\mu_{K}(M) \leq \eta_{K}(M) \leq \nu_{K}(M) \leq \bar{\sigma}(M) .
$$

The following theorem is a direct consequence of (1.4) and Proposition 4.1.

Theorem 4.1. For any matrix $M$ and associated structure $K$,

$$
\begin{aligned}
\mu_{K}(M) & \leq \inf _{D \in D_{K}} \eta_{K}\left(D M D^{-1}\right) \\
& \leq \inf _{D \in D_{K}} \nu_{K}\left(D M D^{-1}\right) \\
& \leq \inf _{D \in D_{K}} \sigma\left(D M D^{-1}\right) .
\end{aligned}
$$

Theorem 4.1 gives two upper bounds which are less conservative than inf $D \in D_{K} \bar{\sigma}\left(D M D^{-1}\right)$. However, since $\max _{K\left(D M D^{-1}\right)}\left\|D M D^{-1} x\right\|$ may have local maxima that are not global, attempts to evaluate $\eta_{K}\left(D M D^{-1}\right)$ may yield strict lower bounds on this quantity and this may result in underestimation of $\mu_{K}(M)$. Fortunately, the second upper bound in (4.2) does not suffer from this shortcoming. In view of the definition of $\nu_{K}(M)$, one encounters the optimization problem

$$
\inf _{D \in D_{K}} \inf _{G \in \mathcal{G}_{K}} F(D, G)
$$

where $F: D_{X} \times \mathcal{G}_{K} \rightarrow \mathbf{R}$ is defined by

$$
F(D, G)=\bar{\lambda}\left[M_{D}^{H} M_{D}+\mathrm{j}\left(G M_{D}-M_{D}^{B} G\right)\right]
$$

with $M_{D}=D M D^{-1}$. Thus, for any $D \in D_{K}, G \in$ $G_{K}, \sqrt{F(D, G)}$ is an upper bound for $\mu_{K}(M)$ (unless $F(D, G)<0$, in which case $\left.\mu_{K}(M)=0\right)$. The infimum can be approached at reasonable CPU cost if $F$ is quasiconvex jointly in $(D, G)$.

Proposition 4.2. $F(D, G)$ is quasi-convex in $D$ and convex in $G$.

Numerical investigation suggests the following.

Conjecture 4.1. $F$ is quasi-convex jointly in $(D, G)$.

Finally, there is an open class of problems for which the new upper bound is equal to $\mu_{K}(M)$.

Theorem 4.2. Suppose that the infimum in (4.3) is achieved and the corresponding largest eigenvalue is simple. Then

$$
\begin{aligned}
\mu_{X}(M) & =\inf _{D \in D_{K}} \eta_{X}\left(D M D^{-1}\right) \\
& =\max \left\{0, \inf _{D \in D_{K}} \nu_{K}\left(D M D^{-1}\right)\right\} .
\end{aligned}
$$

5. Correspondence with the Linear Fractional Transformation Approach

In [2], it is shown that in the case $k_{q}=1, q=$ $1, \ldots, m_{r}$, given $\alpha>0$, a sufficient condition to insure that $\mu_{K}(M) \leq \alpha$ is that,

$$
\inf _{D \in D_{K}} \inf _{C \in \mathcal{C}_{X}} \bar{\sigma}\left[j C+\left(I-C^{2}\right)^{1 / 2} D\left(\frac{M}{\alpha}\right) D^{-1}\right] \leq 1
$$


where

$$
C_{K}=\left\{\operatorname{diag}\left(c_{1}, c_{2}, \ldots, c_{m_{r}}, 0, \ldots, 0\right): c_{i} \in[-1,1]\right\}
$$

Using the bijections from $(-1,1)$ to $\mathbf{R}$

$$
c \rightarrow g=\frac{c}{\sqrt{1-c^{2}}}
$$

it is easily checked that condition (5.1) is equivalent to

$$
\inf _{D \in D_{\times}} \inf _{G \in \mathcal{G}_{\times}} \bar{\sigma}\left[\left(D\left(\frac{M}{\alpha}\right) D^{-1}+j G\right)\left(I+G^{2}\right)^{-1 / 2}\right] \leq 1 .
$$

Furthermore, the following proposition, which can be verified with simple algebra, connects (5.2) with (4.3).

Proposition 5.1. (5.2) holds if, and only if,

$$
\inf _{D \in D_{K}} \inf _{G \in \mathcal{G}_{K}} F(D, G) \leq \alpha^{2}
$$

It can be checked that this equivalence still holds in the case of repeated real scalar blocks. Thus, (4.3) and (5.1) are still equivalent, provided $C$ consists now of Hermitian block diagonal matrices in the place of the scalars. The advantage of (4.3) is that it has much better computational properties. The characterizations in (5.1) and (5.2) may still be useful in the context of $\mu$-synthesis, which uses the upper bounds and $H_{\infty}$ optimal control to synthesize controllers. This is under investigation.

\section{Acknowledgements}

The authors wish to thank Drs. E. Zafiriou and A.K. Packard for numerous discussions.

The work of the first two authors was supported by the National Science Foundation under Grants No. DMC-84-51515 and CDR-85-00108. The work of the third author was supported by NASA, NSF, ONR, and AFOSR.

\section{References}

[1] J.C. Doyle, "Analysis of Feedback Systems with Structured Uncertainties," Proc. IEE-D 129 (1982), 242-250.

[2] J.C. Doyle, "Structured Uncertainty in Control System Design," Proceedings of the 24th IEEE Conference on Decision and Control, Fort Lauderdale, Florida (December 1985).

[3] O. Yaniv \& I. M. Horowitz, "A Quantitative Design Method for MMO Linear Feedback Systems Having Uncertain Plants," Internat. J. Control 43 (1986), 401-421.

[4] J. Ackermann, Sampled-Data Control Systems, SpringerVerlag, New York, Heidelberg, Berlin, 1985.

[5] R.R.E. de Gaston \& M.G. Safonov, "Exact Calculation of the Multiloop Stability Margin," IEEE Trans. Automat. Control AC-33 (1988), 156-171.
[6] A. Sideris \& R.R.E de Gaston, "Multivariable Stability Margin Calculation with Uncertain Correlated $\mathrm{Pa}$ rameters," Proceedings of the 1987 American Control Conference, Minneapolis, Minnesota (June, 1987).

[7] A. Sideris \& R.S. Sánchez Peña, "Fast Computation of the Multivariable Stability Margin for Real Interrelated Uncertain Parameters," Proceedings of the 1988 American Control Conference, Atlanta, Georgia (June, 1988).

[8] V.L. Kharitonov, "Asymptotic Stability of an Equilibrium Position of a Family of Systems of Linear Differential Equations," Differentsial'nye Uraveniya 14 (1978), 2086-2088, Differential Equations 14 (1979), 1483-1485.

[9] A.C. Bartlett, C.V. Hollot \& H. Lin, "Root Locations of an Entire Polytope of Polynomials: It Suffices to Check the Edges," Math. of Control, Signals and Systems 1 (1988), 61-71.

[10] M. Fu \& B.R. Barmish, "Stability of Convex and Linear Combinations of Polynomials and Matrices Arising in Robustness Problems," Proc. of the 1987 Conf. on Information Sciences and Systems, Baltimore, Maryland (1987).

[11] I.R. Petersen, “A New Extension to Kharitonov's Theorem," Proc. of the 26th IEEE Conf. on Decision and Control, Los Angeles, California (December 1987).

[12] A.K. Packard, "What's New with $\mu$ : Structured Uncertainty in Multivariable Control," University of California, $\mathrm{PhD}$ Thesis, Berkeley, California, 1988.

[13] M.K.H. Fan, "An Algorithm to Compute the Structured Singular Value," Systems Research Center, University of Maryland, College Park, Technical Report TR-86-8, 1986.

[14] M.K.H. Fan \& A.L. Tits, "Characterization and Efficient Computation of the Structured Singular Value," IEEE Trans. Automat. Control AC-31 (1986), 734743.

[15] M.K.H. Fan \& A.L. Tits, "m-Form Numerical Range and the Computation of the Structured Singular Value," IEEE Trans. Automat. Control AC-33 (1988), 284-289.

[16] S. Friedland \& R. Loewy, "Subspaces of Symmetric Matrices Containing Matrices with a Multiple First Eigenvalue," Pacific J. Math.62 (1976), 389-399.

[17] Y.-H. Au-Yeung \& Y.-T. Poon, "A Remark on the Convexity and Positive Definiteness Concerning Hermitian matrices," Southeast Asian Bull. Math. 3 (1979), 85-92.

[18] M.K.H. Fan \& A.L. Tits, "On the Generalized Numerical Range," Linear and Multilinear Algebra 21 (1987), 313-320. 